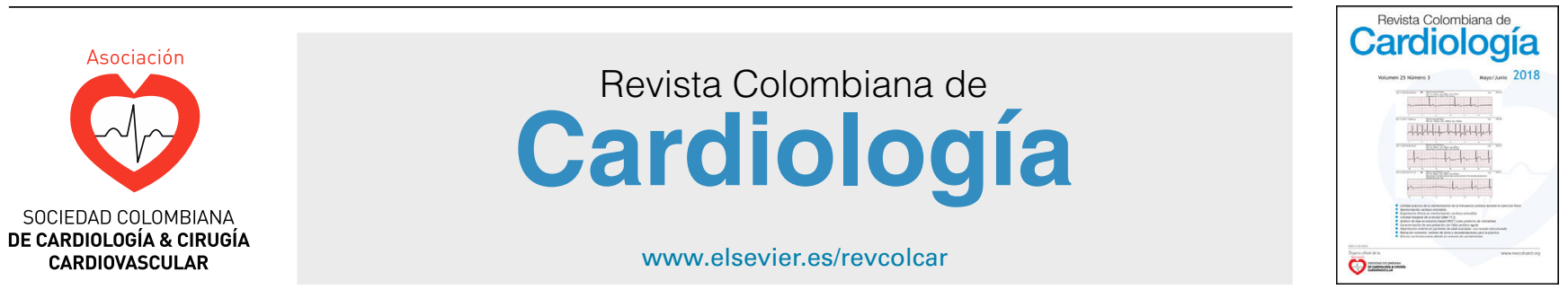

CARDIOLOGÍA DEL ADULTO - REVISIÓN DE TEMAS

\title{
Verdades y controversias de la anticoagulación en fibrilación auricular no valvular
}

\section{Jaime Alberto Gómez-Rosero ${ }^{\mathrm{a}}$ y Elsy Cristina Sierra-Vargas $\mathrm{a,b,c,*}$}

\author{
a Medicina interna, Universidad de Antioquia, Medellín, Colombia \\ b Universidad CES, Medellín, Colombia \\ c Universidad Cooperativa de Colombia, Medellín, Colombia
}

Recibido el 8 de abril de 2019; aceptado el 14 de julio de 2019

Disponible en Internet el 11 October 2019

\section{PALABRAS CLAVE}

Fibrilación auricular;

Ataque

cerebrovascular

isquémico;

Anticoagulantes

orales de acción

directa;

Warfarina

\section{KEYWORDS}

Atrial fibrillation;

Ischaemic cerebrovascular accident; Direct action oral anticoagulants;

Warfarin

\begin{abstract}
Resumen La fibrilación auricular es la arritmia de significado clínico más frecuente a la que médicos generales y especialistas se pueden ver enfrentados. Esta enfermedad aumenta cinco veces el riesgo de ataque cerebrovascular. La evidencia actual favorece el uso de anticoagulantes orales de acción directa sobre warfarina para la prevención de eventos cardioembólicos en pacientes con fibrilación auricular no valvular, en ausencia de contraindicaciones. En este artículo se discutirán a modo de preguntas y respuestas los aspectos más importantes, así como algunas controversias acerca de la anticoagulación en fibrilación auricular no valvular.

(c) 2019 Sociedad Colombiana de Cardiología y Cirugía Cardiovascular. Publicado por Elsevier España, S.L.U. Este es un artículo Open Access bajo la licencia CC BY-NC-ND (http:// creativecommons.org/licenses/by-nc-nd/4.0/).
\end{abstract}

\section{Truths and controversies about coagulation in non-valvular atrial fibrillation}

Abstract Atrial fibrillation is the most common clinically significant arrhythmia that may be encountered by general and specialist doctors. This disorder increases the risk of a cerebrovascular accident by five times. The current evidence prefers the use of direct action oral anticoagulants over warfarin for the prevention of cardioembolic events in patients with nonvalvular atrial fibrillation in the absence of contraindications. Using a series of questions and answers, the most important aspects will be discussed in this article, as well as some controversies about anticoagulation in non-valvular atrial fibrillation.

(c) 2019 Sociedad Colombiana de Cardiología y Cirugía Cardiovascular. Published by Elsevier España, S.L.U. This is an open access article under the CC BY-NC-ND license (http:// creativecommons.org/licenses/by-nc-nd/4.0/).

\footnotetext{
* Autor para correspondencia.

Correo electrónico: sierravargasmd@gmail.com (E.C. Sierra-Vargas).
} 


\section{Introducción}

La incidencia de ataque cerebrovascular en Estados Unidos es de aproximadamente 795.000 casos cada año, de los cuales 610.000 son primer episodio y 185.000 tienen antecedente de ataque cerebrovascular previo ${ }^{1}$. Es la segunda causa de muerte y la tercera causa de discapacidad en el mundo ${ }^{2}$. En Colombia, su incidencia en el año 2010 fue de 97,3 por 100.000 personas/año, similar a lo reportado en $1990(97,4 \text { por } 100.000 \text { persona/año })^{3}$, cifras que cuestionan el alcance de las estrategias de prevención y control de los factores de riesgo cardiocerebrovasculares en nuestra población. Por su parte, la mortalidad pasó de 37,7 por 100.000 personas/año en 1990 a 28,5 por 100.000 personas/año en $2010^{3}$, probablemente en relación con los avances en el diagnóstico, el manejo agudo del ataque cerebrovascular y la rehabilitación integral.

Cerca del $80 \%$ de los ataques cerebrovasculares son de origen isquémico; de estos, un 20 a un $30 \%$ son atribuibles a fibrilación auricular diagnosticada antes, durante o después del evento ${ }^{4}$. El riesgo de ataque cerebrovascular (u otro evento embólico) no es igual en todos los pacientes con fibrilación auricular y depende de varios factores que han sido incorporados en las conocidas escalas de riesgo ${ }^{5}$. La tasa de eventos tromboembólicos en pacientes con fibrilación auricular que no reciben anticoagulación puede ir desde $0 \%$ hasta

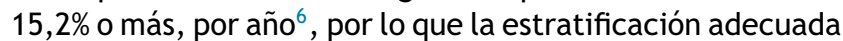
es fundamental para definir qué pacientes se benefician de anticoagulantes orales.

\section{¿Qué características tiene el ataque cerebrovascular por fibrilación auricular?}

La afectación territorial del ataque cerebrovascular por fibrilación auricular parece ser distinta al ataque cerebrovascular de origen carotídeo. En un análisis retrospectivo se encontró que en pacientes con fibrilación auricular los eventos isquémicos retinianos fueron infrecuentes comparados con los eventos cerebrales (relación 1:32), mientras que en pacientes con estenosis carotídea significativa los eventos retinianos ocurrieron la mitad de las veces que los hemisféricos ${ }^{7}$. De otro lado, cuando se compara con todos los ataques cerebrovasculares que no son producidos por fibrilación auricular, la mayoría de los estudios reporta que la mortalidad a 30 días y a 3 meses es mayor, la estancia hospitalaria es más prolongada y los índices de funcionalidad neurológica a los 3, 6 y 12 meses son peores en los ataques cerebrovasculares originados por fibrilación auricular ${ }^{8,9}$.

Se ha encontrado que los pacientes con fibrilación auricular tienen dos a tres veces más riesgo de infartos cerebrales silentes comparados con los pacientes en ritmo sinusal, independiente del tipo de fibrilación auricular (paroxística vs. permanente $)^{10}$. Estos infartos silentes se han asociado con un aumento en el riesgo de ataque cerebrovascular sintomático y demencia ${ }^{11,12}$, por lo que es razonable pensar que este subgrupo de pacientes se puede beneficiar de la anticoagulación. No obstante, es importante advertir que: 1) la definición de infarto cerebral silente varía entre los diferentes estudios y su detección depende de la técnica de imagen utilizada (siendo más sensible, pero más costosa y menos disponible, la resonancia que la tomografía $)^{10}$, y 2 ) el impacto de la anticoagulación en la prevención del infarto cerebral silente es incierto porque la evidencia disponible es muy escasa y de limitado rigor metodológico ${ }^{13,14}$. Con base en lo anterior se sugiere no realizar neuroimagen de rutina a pacientes con diagnóstico de fibrilación auricular que no tengan síntomas o signos neurológicos. En caso de documentar de forma incidental un infarto cerebral silente en un paciente con fibrilación auricular nos inclinamos a favor de la anticoagulación.

\section{¿Qué escala debo usar para clasificar el riesgo de eventos tromboembólicos y el riesgo de sangrado?}

Existes varios puntajes para estratificar el riesgo de embolia en pacientes con fibrilación auricular; entre los más conocidos están: $\mathrm{CHADS}_{2}, \mathrm{CHA}_{2} \mathrm{DS}_{2} \mathrm{VASc}$, ATRIA, Framingham, $\mathrm{R}_{2} \mathrm{CHADS}_{2}, \mathrm{CHA}_{2} \mathrm{DS}_{2} \mathrm{VASc}-\mathrm{R}, \mathrm{AFI}$ y SPAF ${ }^{15-21}$. Estas escalas son formas aceptables de aproximarse al riesgo embólico, pero no abarcan todo el espectro clínico de la enfermedad, ya que pueden dejar por fuera individuos que eventualmente se beneficien de la anticoagulación. En la actualidad se recomienda utilizar la $\mathrm{CHA}_{2} \mathrm{DS}_{2}$ VASc porque, comparada con la $\mathrm{CHADS}_{2}$, ha demostrado mejor capacidad para discriminar los pacientes de muy bajo riesgo tromboembólico y un desempeño igual o superior para predecir los de alto riesgo $^{16}$.

Las guías recomiendan iniciar anticoagulación si la $\mathrm{CHA}_{2} \mathrm{DS}_{2}$ VASc es $\geq 2$ en hombres $\mathrm{y} \geq 3$ en mujeres, mientras que si la $\mathrm{CHA}_{2} \mathrm{DS}_{2} \mathrm{VASc}$ es de 0 en hombres y 1 en mujeres se recomienda no anticoagular ${ }^{4,22}$. Con un nivel de evidencia más bajo, recomiendan que la anticoagulación pudiera considerarse en hombres con $\mathrm{CHA}_{2} \mathrm{DS}_{2}$ VASc de 1 y mujeres con $\mathrm{CHA}_{2} \mathrm{DS}_{2} \mathrm{VASc}$ de 2 . En esta población el riesgo de ataque cerebrovascular es heterogéneo y depende del "peso" de cada variable (por ejemplo, el riesgo de ataque cerebrovascular es mayor para el rango de edad entre 65 a 74 años que para el antecedente de hipertensión arterial), lo cual hace que el beneficio neto de la anticoagulación sea distinto entre un paciente y otro ${ }^{23}$. La decisión de anticoagular a este grupo de riesgo intermedio puede refinarse con la consideración de otras variables que aumentan el riesgo embólico, como la obesidad, el aumento del tamaño de la aurícula izquierda o la disfunción renal. La medición de troponina o péptidos natriuréticos también puede ayudar a la toma de decisiones ${ }^{24}$.

En cuanto al riesgo de sangrado, las principales escalas son HEMORR 2 HAGES, HAS-BLED, ATRIA y ORBIT ${ }^{25-28}$. La que mejor predice sangrado intracraneal es HAS-BLED. Pacientes con HAS-BLED $\geq 3$ puntos clasifican como alto riesgo. Estas escalas se deben emplear con el propósito de identificar y corregir los factores modificables que predisponen a eventos hemorrágicos, pero no constituyen una herramienta para no iniciar o suspender la anticoagulación en el paciente que tiene indicación de recibirla ${ }^{29}$.

\section{¿Qué anticoagulante debe ser primera línea?}

Existen dos grupos de anticoagulantes orales: los antagonistas de la vitamina $\mathrm{K}$ cuyo prototipo es la warfarina, $\mathrm{y}$ 
los anticoagulantes orales de acción directa (DOAC, por la sigla en inglés de direct oral anticoagulants), de los cuales están aceptados para el manejo de la fibrilación auricular dabigatrán, rivaroxabán, apixabán y edoxabán.

Warfarina es el único medicamento aprobado para pacientes con fibrilación auricular valvular. Aunque, comparada con los DOAC, su costo anual es menor ${ }^{30}$, tiene más años en el mercado y mayor disponibilidad, la warfarina parece no ser costoefectiva a largo plazo ${ }^{31}$, tiene una ventana terapéutica estrecha, más interacciones farmacológicas y alimentarias, necesita monitorización periódica, tiene mayor riesgo de sangrado y si el tiempo en rango terapéutico (TRT) es menor a $65 \%$ tiene también más riesgo de complicaciones trombóticas ${ }^{32}$. Por consiguiente en la actualidad, en pacientes con fibrilación auricular no valvular que no tengan contraindicaciones, la primera línea es un $\mathrm{DOAC}^{4,22}$. No obstante, la warfarina sigue siendo uno de los anticoagulantes más utilizados en Colombia, especialmente en la población en la que no es posible garantizar el suministro de los DOAC por parte del asegurador. Una de las grandes dificultades con warfarina es garantizar un TRT óptimo, ya que incluso en el escenario ideal de los ensayos clínicos controlados que sirvieron para la aprobación de los DOAC, el TRT fue 55 a 64\%. En los únicos dos estudios realizados en clínicas de anticoagulación del país, el TRT fue $54 \%{ }^{33,34}$, porcentaje que evidencia la realidad del control en nuestra población. La aplicación del modelo clínico SAMe-TT2R2 (sexo femenino, edad menor a 60 años, comorbilidades, interacciones farmacológicas, tabaquismo -2 puntos - , raza no blanca -2 puntos - ) puede ayudar a predecir quienes tienen más riesgo de una anticoagulación subóptima con warfarina: un puntaje mayor o igual a 2 se correlaciona con un tiempo en rango terapéutico por debajo del $65 \%^{35}$. Cabe anotar que este modelo no ha sido validado en población latina.

Respecto a qué DOAC escoger, todos han demostrado prevenir eventos tromboembólicos en pacientes con fibrilación auricular no valvular, así que, en principio, cualquiera podría ser utilizado. Sin embargo, teniendo en cuenta los resultados de eficacia y seguridad, en algunos escenarios se favorece el uso de un DOAC sobre otro. Nuestras recomendaciones son $^{36-38}$ :

- Paciente con alto riesgo de sangrado gastrointestinal: iniciar apixabán (se prefiere) o dabigatrán $110 \mathrm{mg}$. La razón: rivaroxabán, dabigatrán $150 \mathrm{mg}$ y edoxabán aumentaron de forma significativa el sangrado gastrointestinal en sus respectivos estudios.

- Paciente con alto riesgo de sangrado mayor: las opciones son -en orden de preferencia- apixabán, dabigatrán $110 \mathrm{mg}$ y edoxabán. La razón: son los DOAC con las tasas más bajas de sangrado mayor.

- Ataque cerebrovascular que recurre a pesar de estar en manejo con warfarina (con INR en metas): iniciar dabigatrán $150 \mathrm{mg}$. La razón: comparado con warfarina, la reducción del riesgo de ataque cerebrovascular/embolia sistémica fue del $34 \%$ para dabigatrán $150 \mathrm{mg}$ (estudio RE-LY), del 21\% para apixabán (estudio ARISTOTLE), del 13\% para edoxabán (estudio ENGAGE AF-TIMI), del 12\% para rivaroxabán (estudio ROCKET AF) y del 9\% para dabigatrán $110 \mathrm{mg}$ (estudio RE-LY). Cabe señalar que en los ensayos clínicos controlados dabigatrán fue el único DOAC que demostró reducir de forma significativa la incidencia de ataque cerebrovascular isquémico versus warfarina; para los demás DOAC la reducción de los eventos cerebrovasculares se dio a expensas del ataque cerebrovascular hemorrágico.

- Paciente con quejas gastrointestinales: iniciar apixabán, rivaroxabán o edoxabán. La razón: dabigatrán puede producir dispepsia y diarrea (incidencia de 11 y 6\%, respectivamente - datos del RE-LY).

- Paciente con $\mathrm{CHA}_{2} \mathrm{DS}_{2}$ VASc de 1 punto (o 2 puntos si es mujer): se prefieren los DOAC con las tasas más bajas de sangrado mayor (apixabán, dabigatrán $110 \mathrm{mg}$ o edoxabán). La razón: en este escenario no hacer daño está por encima de evitar un ataque cerebrovascular.

No se recomienda el uso de antiagregantes (aspirina o clopidogrel) en reemplazo de la anticoagulación, porque tienen similares tasas de sangrado y son menos eficaces para prevenir ataque cerebrovascular que los anticoagulantes orales ${ }^{39,40}$.

\section{¿Qué hacer en los pacientes con alto riesgo hemorrágico?}

Como ya se mencionó, tener un puntaje elevado en una escala de riesgo hemorrágico no contraindica la anticoagulación sino que alerta al médico a una vigilancia más estrecha de su paciente y conduce a un control más estricto de los factores de riesgo modificables que incrementan el riesgo de sangrado, como la hipertensión arterial fuera de metas, un TRT menor a $65 \%$ en pacientes que están con warfarina, el uso concomitante de AINE o antiagregantes plaquetarios, el consumo de alcohol y la anemia.

\section{¿Puede un paciente con sangrado intracraneal o gastrointestinal continuar anticoagulado?}

La hemorragia intracerebral afecta a menos del $1 \%$ de los pacientes anticoagulados cada año; sin embargo, sus consecuencias pueden ser devastadoras. Los pacientes anticoagulados por fibrilación auricular que presenten sangrado intracraneal deben ser evaluados por un equipo multidisciplinario que incluya cardiología, neurología y neurocirugía, y si el balance riesgo/beneficio favorece la anticoagulación, se recomienda reiniciarla cuatro a ocho semanas después del evento, siempre y cuando los factores precipitantes (uso de antiagregantes, hipertensión arterial mal controlada, entre otros) hayan sido corregidos ${ }^{4}$. En estos casos se deben considerar los medicamentos que en los estudios han mostrado menor tasa de sangrado intracraneal (dabigatrán, apixabán y edoxabán $)^{37}$. En sangrados intracraneales asociados a warfarina el reinicio de la anticoagulación disminuye el riesgo tromboembólico y de muerte, sin aumentar significativamente los eventos hemorrágicos. Hay dos condiciones que se asocian con alto riesgo de resangrado: la angiopatía amiloide y el hematoma subdural espontáneo, en estos pacientes la decisión de reiniciar la anticoagulación representa un gran reto, y el mejor momento para hacerlo deberá ser acordado por los miembros del equipo multidisciplinario ${ }^{41}$. 
En pacientes con sangrado gastrointestinal el panorama es más claro: una vez controlada la fuente del sangrado, parece seguro reiniciar la anticoagulación al alta hospitalaria. Esta conducta se ha asociado con menores tasas de trombosis, sin aumento del resangrado a 90 días $^{41}$.

\section{¿Qué paso debo seguir en un paciente anticoagulado con warfarina en rango terapéutico que presenta un ataque cerebrovascular isquémico?}

Cuando un paciente tiene un evento cerebrovascular mientras está en manejo con warfarina, lo más probable es que el INR sea menor a 2 o que el TRT sea menor al 65\%. En ellos la warfarina puede seguir siendo una opción siempre y cuando se garantice INR en metas. De otro lado, en pacientes que presentan el ataque cerebrovascular estando en rango terapéutico y que se presume origen cardioembólico, no hay ensayos clínicos que evalúen qué estrategia seguir y queda en manos del equipo tratante tomar la decisión más apropiada. En estos casos preferimos cambiar a dabigatrán $150 \mathrm{mg}$ cada 12 horas, que como se mencionó es el único DOAC que ha demostrado reducir de manera significativa el ataque cerebrovascular isquémico frente a la warfarina ${ }^{42}$. Otra alternativa sería cambiar a apixabán teniendo en cuenta los resultados del estudio ARISTOPHANES, el más grande a la fecha en evaluar el comportamiento de los DOAC en el "mundo real". Este estudio incluyó 321.182 pacientes con fibrilación auricular no valvular y comparó, mediante el puntaje de propensión, warfarina con apixabán, dabigatrán y rivaroxabán. Los DOAC tuvieron las tasas más bajas de ataque cerebrovascular/embolia sistémica, con una reducción, versus warfarina, del 39\% para apixabán, $25 \%$ para rivaroxabán y $20 \%$ para dabigatrán ${ }^{43}$.

\section{¿Qué hacer si el ataque cerebrovascular se presenta en un paciente anticoagulado con los nuevos anticoagulantes orales?}

En este escenario hay grandes vacíos en la evidencia y la decisión deberá ser tomada por un equipo multidisciplinario. Lo primero es evaluar la adherencia al tratamiento y que la dosificación sea la correcta; una vez asegurado esto, las opciones de manejo incluyen adicionar un antiagregante plaquetario, cambiar a warfarina o cerrar la auriculilla izquierda. Cabe recordar que los DOAC reducen el riesgo de eventos tromboembólicos pero no lo evitan por completo; de hecho, en las condiciones ideales de los grandes ensayos clínicos, la frecuencia de ataque cerebrovascular/embolia sistémica fue de 1,1 a 2,1\% por año.

\section{¿Es segura y eficaz la anticoagulación en falla renal terminal?}

Los pacientes con fibrilación auricular y enfermedad renal crónica avanzada (depuración de creatinina menor a $30 \mathrm{ml} / \mathrm{min}$ ) tienen mayor riesgo de complicaciones tromboembólicas y hemorrágicas, comparados con pacientes sin falla renal ${ }^{44}$. Infortunadamente, no hay evidencia sólida acerca del beneficio y la seguridad de la anticoagulación en pacientes con fibrilación auricular y enfermedad renal crónica avanzada porque esta población ha sido excluida de los ensayos clínicos controlados ${ }^{45}$. En pacientes con enfermedad renal crónica terminal (depuración de creatinina $<15 \mathrm{ml} / \mathrm{min}$ o en diálisis) la warfarina ha sido el anticoagulante de elección; sin embargo, los resultados de los estudios observacionales son conflictivos, ya que algunos reportan un aumento en el riesgo de sangrado e incluso de ataque cerebrovascular ${ }^{46-48}$. La evidencia con los DOAC es aún más escasa. Se sabe que la concentración sérica de estos medicamentos aumenta significativamente en pacientes con enfermedad renal crónica terminal, poniéndolos en alto riesgo de sangrado. En una cohorte de pacientes con fibrilación auricular en hemodiálisis el uso off-label de dabigatrán y rivaroxabán se asoció con un aumento en el riesgo de sangrado mayor, comparado con warfarina ${ }^{49}$. Estos resultados contrastan con los de un estudio retrospectivo reciente de apixabán versus warfarina en pacientes con fibrilación auricular en diálisis, que encontró que apixabán disminuyó de forma significativa el riesgo de sangrado mayor, y en el subgrupo de pacientes con dosis estándar de apixabán $(5 \mathrm{mg}$ cada 12 horas) se redujo además el riesgo de ataque cerebrovascular y muerte ${ }^{50}$. Este estudio sirvió para que en 2019 la guía americana pusiera a apixabán, junto con warfarina, como los únicos anticoagulantes que pueden considerarse (recomendación IIb) en pacientes con fibrilación auricular y enfermedad renal crónica terminal.

En nuestra opinión, existe mucha incertidumbre sobre la seguridad y eficacia de la anticoagulación en esta población, así que el médico deberá hacer un ejercicio reflexivo del balance riesgo/beneficio en cada caso. Se espera que el ensayo clínico AXADIA (apixabán versus warfarina en pacientes con fibrilación auricular en diálisis) que se encuentra en fase de reclutamiento, aporte más elementos para responder esta pregunta ${ }^{51}$.

\section{¿Quién es candidato al cierre de la auriculilla?}

La auriculilla izquierda es el sitio donde se forman los trombos en más del $90 \%$ de los pacientes con fibrilación auricular no valvular. El cierre percutáneo de la auriculilla izquierda con el dispositivo Watchman fue evaluado en los estudios PROTECT AF y PREVAIL, cuyos resultados sirvieron para que el procedimiento fuera aprobado por la Administración de Drogas y Medicamentos. El PROTECT AF fue un ensayo clínico de no inferioridad que comparó el uso del dispositivo Watchman con warfarina en pacientes con fibrilación auricular no valvular. Después del procedimiento los pacientes fueron tratados con warfarina durante 45 días y luego recibieron clopidogrel más aspirina por 6 meses, seguido de aspirina por tiempo indefinido. El desenlace primario fue el compuesto de ataque cerebrovascular, embolia sistémica y muerte cardiovascular, en tanto que el desenlace de seguridad fue un compuesto de sangrado mayor y complicaciones derivadas del procedimiento. Después de un seguimiento medio de 18 meses, la tasa de eventos del desenlace primario fue similar en ambos grupos, concluyendo que el cierre de la auriculilla es no inferior a warfarina para la prevención de ataque cerebrovascular; sin embargo, hubo una frecuencia mayor de eventos adversos (derrame pericárdico $-70 \%$ de 
los casos requirieron pericardiocentesis-, sangrado mayor y ataque cerebrovascular isquémico periprocedimiento) en el grupo de la intervención ${ }^{52}$. Cinco años después se publicó el estudio PREVAIL, con metodología similar al PROTECT AF. A los 18 meses de seguimiento, el desenlace de ataque cerebrovascular, embolia sistémica y muerte cardiovascular no cumplió el criterio de no inferioridad, pero el desenlace de ataque cerebrovascular o embolia sistémica mayor a 7 días posprocedimiento fue no inferior a warfarina. Los eventos adversos disminuyeron de forma significativa, lo cual sugiere que la seguridad de la intervención mejoró con la experiencia adquirida ${ }^{53}$. Estos ensayos clínicos tienen tres grandes limitaciones: la primera es que excluyeron a los pacientes que tuvieran contraindicación absoluta para recibir warfarina, por lo que la eficacia y seguridad del procedimiento en esta población son inciertas; la segunda es que los pacientes deben recibir antiagregación de forma indefinida, con el riesgo de sangrado que eso conlleva; y la tercera es que no se comparó el dispositivo Watchman con los DOAC.

Aunque el cierre percutáneo de la auriculilla izquierda es comparable con warfarina en la prevención de ataque cerebrovascular y tiene menos riesgo de sangrado ${ }^{54}$, no es una terapia de primera línea porque es invasiva y no está exenta de eventos adversos. Las guías actuales mencionan que puede considerarse (recomendación IIb) para pacientes con contraindicación a largo plazo para la anticoagulación por predisposición a sangrado o por pobre tolerancia/adherencia al medicamento ${ }^{4,22}$.

\section{Conclusión}

La fibrilación auricular es una causa frecuente de ataque cerebrovascular isquémico, desenlace con alta carga de morbimortalidad. Por consiguiente, es fundamental identificar la población que se beneficia de anticoagulación con miras a disminuir el riesgo de eventos cardioembólicos. A la fecha, los anticoagulantes orales directos son la primera línea en el tratamiento de los pacientes con fibrilación auricular no valvular. De los ensayos clínicos más representativos hemos obtenido las claves para individualizar la prescripción de la anticoagulación, por lo que recomendamos evaluar las condiciones que favorecen el uso de un medicamento sobre otro. La evidencia científica se queda corta acerca de qué hacer en casos en los que la terapia estándar falla, y es aquí cuando se debe recurrir al juicio clínico y al apoyo de un grupo multidisciplinario con el fin de tomar la mejor decisión.

\section{Financiación}

Ninguna.

\section{Conflictos de interés}

Ninguno.

\section{Bibliografía}

1. Benjamin EJ, Blaha MJ, Chiuve SE, Cushman M, Das SR, Deo $R$, et al. American Heart Association Statistics Committee and Stroke Statistics Subcommittee. Heart Disease and Stroke Statistics-2017 Update: A Report From the American Heart Association. Circulation. 2017;135:e146-603.

2. Lozano R, Naghavi M, Foreman K, Lim S, Shibuya K, Aboyans $V$, et al. Global and regional mortality from 235 causes of death for 20 age groups in 1990 and 2010: a systematic analysis for the Global Burden of Disease Study 2010. Lancet. 2012;380:2095-128.

3. Krishnamurthi RV, Feigin VL, Forouzanfar MH, Mensah GA, Connor M, Bennett DA, et al. Global Burden of Diseases, Injuries. Risk Factors Study 2010 (GBD 2010); GBD Stroke Experts Group. Global and regional burden of first-ever ischaemic and haemorrhagic stroke during 1990-2010: findings from the Global Burden of Disease Study 2010. Lancet Glob Health. 2013;1:e259-81.

4. Kirchhof P, Benussi S, Kotecha D, Ahlsson A, Atar D, Casadei B, et al., 2016 ESC Guidelines for the management of atrial fibrillation developed in collaboration with EACTS. Eur Heart J. 2016;37:2893-962.

5. Lip GY. Can we predict stroke in atrial fibrillation? Clin Cardiol. 2012;35 Suppl 1:21-7.

6. Lip GY, Halperin JL. Improving stroke risk stratification in atrial fibrillation. Am J Med. 2010;123:484-8.

7. Anderson DC, Kappelle LJ, Eliasziw M, Babikian VL, Pearce LA, Barnett HJ. Occurrence of hemispheric and retinal ischemia in atrial fibrillation compared with carotid stenosis. Stroke. 2002;33:1963.

8. Lin HJ, Wolf PA, Kelly-Hayes M, Beiser AS, Kase CS, Benjamin EJ, D’Agostino RB. Stroke severity in atrial fibrillation. The Framingham Stud. Stroke. 1996;27:1760.

9. Jørgensen HS, Nakayama H, Reith J, Raaschou HO, Olsen TS. Acute stroke with atrial fibrillation. The Copenhagen Stroke Study. Stroke. 1996;27:1765.

10. Kalantarian S, Ay H, Gollub RL, Lee H, Retzepi K, Mansour M, Ruskin JN. Association between atrial fibrillation and silent cerebral infarctions: a systematic review and meta-analysis. Ann Intern Med. 2014;161:650-8.

11. Vermeer SE, Hollander M, van Dijk EJ, Hofman A, Koudstaal PJ, Breteler MM. Silent brain infarcts and white matter lesions increase stroke risk in the general population: the Rotterdam Scan Study. Stroke. 2003;34:1126-9.

12. Vermeer SE, Prins ND, den Heijer T, Hofman A, Koudstaal PJ, Breteler MM. Silent brain infarcts and the risk of dementia and cognitive decline. N Engl J Med. 2003;348:1215-22.

13. Ezekowitz MD, James KE, Nazarian SM, Davenport J, Broderick JP, Gupta SR, et al. Silent cerebral infarction in patients with nonrheumatic atrial fibrillation. The Veterans Affairs Stroke Prevention in Nonrheumatic Atrial Fibrillation Investigators. Circulation. 1995;92:2178-82.

14. Hahne K, Mönnig G, Samol A. Atrial fibrillation and silent stroke: links, risks, and challenges. Vasc Health Risk Manag. 2016;12:65-74.

15. Gage BF, Waterman AD, Shannon W, Boechler M, Rich MW, Radford MJ. Validation of clinical classification schemes for predicting stroke: results from the National Registry of Atrial Fibrillation. JAMA. 2001;285:2864-70.

16. Lip GY, Nieuwlaat R, Pisters R, Lane DA, Crijns HJ. Refining clinical risk stratification for predicting stroke and thromboembolism in atrial fibrillation using a novel risk factor-based approach: the euro heart survey on atrial fibrillation. Chest. 2010;137:263-72.

17. Wang TJ, Massaro JM, Levy D, Vasan RS, Wolf PA, D’Agostino $\mathrm{RB}$, et al. A risk score for predicting stroke or death in individuals with new-onset atrial fibrillation in the community: the Framingham Heart Study. JAMA. 2003;290:1049-56.

18. Piccini JP, Stevens SR, Chang Y, Singer DE, Fox KA, Califf $\mathrm{RM}$, et al. Renal dysfunction as a predictor of stroke and systemic embolism in patients with nonvalvular atrial fibrillation: validation of the $R(2) \operatorname{CHADS}(2)$ index in the ROCKET AF 
(Rivaroxaban Once-daily, oral, direct factor Xa inhibition Compared with vitamin $\mathrm{K}$ antagonism for prevention of stroke and Embolism Trial in Atrial Fibrillation) and ATRIA (AnTicoagulation and Risk factors In Atrial fibrillation) study cohorts. Circulation. 2013;127:224-32.

19. Singer DE, Chang Y, Borowsky LH, Fang MC, Pomernacki NK, Udaltsova N, et al. A new risk scheme to predict ischemic stroke and other thromboembolism in atrial fibrillation: the ATRIA study stroke risk score. J Am Heart Assoc. 2013;2:e000250.

20. Kabra R, Girotra S, Vaughan Sarrazin M. Refining Stroke Prediction in Atrial Fibrillation Patients by Addition of AfricanAmerican Ethnicity to CHA2DS2-VASc Score. J Am Coll Cardiol. 2016;68:461-70.

21. Anderson DC, Halperin JL, Hart RG, McAnulty JH, McBride R, Pearce LA, Sherman DG. Patients with nonvalvular atrial fibrillation at low risk of stroke during treatment with aspirin: Stroke Prevention in Atrial Fibrillation III Study. The SPAF III Writing Committee for the Stroke Prevention in Atrial Fibrillation Investigators. JAMA. 1998 Apr 22-29;279:1273-7.

22. January CT, Wann LS, Calkins H, Chen LY, Cigarroa JE, Cleveland JC Jr, et al. 2019 AHA/ACC/HRS Focused Update of the 2014 AHA/ACC/HRS Guideline for the Management of Patients With Atrial Fibrillation. J Am Coll Cardiol. 2019 Jan 21, pii: S07351097(19)30209-8.

23. Sulzgruber P, Wassmann S, Semb AG, Doehner W, Widimsky P, Gremmel T, et al., Oral anticoagulation in patients with nonvalvular atrial fibrillation and a $\mathrm{CHA}_{2} \mathrm{DS}_{2}$-VASc score of 1 : a current opinion of the European Society of Cardiology Working Group on Cardiovascular Pharmacotherapy and European Society of Cardiology Council on Stroke. Eur Heart J Cardiovasc Pharmacother. 2019;5:171-80.

24. Hijazi Z, Oldgren J, Andersson U, Connolly SJ, Ezekowitz MD, Hohnloser SH, et al. Cardiac biomarkers are associated with an increased risk of stroke and death in patients with atrial fibrillation: a Randomized Evaluation of Long-term Anticoagulation Therapy (RELY) substudy. Circulation. 2012;125: 1605-16.

25. Gage BF, Yan Y, Milligan PE, Waterman AD, Culverhouse R, Rich MW, Radford MJ. Clinical classification schemes for predicting hemorrhage: results from the National Registry of Atrial Fibrillation (NRAF). Am Heart J. 2006;151:713-9.

26. Pisters R, Lane DA, Nieuwlaat R, de Vos CB, Crijns HJ, Lip GY. A novel userfriendly score (HAS-BLED) to assess 1-year risk of major bleeding in patients with atrial fibrillation: the Euro Heart Survey. Chest. 2010;138:1093-100.

27. Fang MC, Go AS, Chang Y, Borowsky LH, Pomernacki NK, Udaltsova N, Singer DE. A new risk scheme to predict warfarin-associated hemorrhage: The ATRIA (Anticoagulation and Risk Factors in Atrial Fibrillation) Study. J Am Coll Cardiol. 2011;58:395-401.

28. O’Brien EC, Simon DN, Thomas LE, Hylek EM, Gersh BJ, Ansell JE, et al. The ORBIT bleeding score: a simple bedside score to assess bleeding risk in atrial fibrillation. Eur Heart J. 2015;36:3258-64.

29. Pérez-Copete J, Esteve-Pastor MA, Roldán V, Valdés $M$, Marín F. Escalas de evaluación del riesgo tromboembólico y hemorrágico en la fibrilación auricular. Rev Esp Cardiol Supl. 2016;16(A):25-32.

30. Machado-Alba J, García-Betancur S, Villegas-Cardona F, MedinaMorales D. Patrones de prescripción de los nuevos anticoagulantes orales y sus costos económicos en Colombia. Rev Colomb Cardiol. 2016;23:277-85.

31. García-Peña A. Evaluación de costo-efectividad de los nuevos anticoagulantes orales en pacientes con fibrilación auricular no valvular. Rev Colomb Cardiol. 2017;24:87-95.

32. Fawzy AM, Lip GY. Pharmacokinetics and Pharmacodynamics of Oral Anticoagulants used in Atrial Fibrillation. Expert Opin Drug Metab Toxicol. 2019;15:381-98.
33. Laverde LP, Gómez SE, Montenegro AC, Lineros A, Wills B, Buitrago Andrés F. Experiencia de una clínica de anticoagulación. Rev Colomb Cardiol. 2015;22:224-30.

34. Miranda H, Osorio S, Giraldo D, Duque J, Ubeimar-Cataño J, Tobón L, et al. Tiempo en rango terapéutico (TRT) en clínica de anticoagulación. Reportes de eventos adversos y factores asociados a bajo TRT. Acta Med Colomb. 2016;41:42-8.

35. Apostolakis S, Sullivan RM, Olshansky B, Lip GY. Factors affecting quality of anticoagulation control among patients with atrial fibrillation on warfarin: the SAMe-TT2R2 score. Chest. 2013;144:1555-63.

36. Hammersley D, Signy M. Navigating the choice of oral anticoagulation therapy for atrial fibrillation in the NOAC era. Ther Adv Chronic Dis. 2017;8:165-76.

37. Farmakis D, Davlouros P, Giamouzis G, Giannakoulas G, Pipilis A, Tsivgoulis G, Parissis J. Direct oral anticoagulants in nonvalvular atrial fibrillation: practical considerations on the choice of agent and dosing. Cardiology. 2018;140:126-32.

38. Ruff CT, Giugliano RP, Braunwald E, Hoffman EB, Deenadayalu N, Ezekowitz MD, et al. Comparison of the efficacy and safety of new oral anticoagulants with warfarin in patients with atrial fibrillation: a meta-analysis of randomised trials. Lancet. 2014;383:955-62.

39. Mant J, Hobbs FD, Fletcher K, Roalfe A, Fitzmaurice D, Lip GY, et al. Warfarin versus aspirin for stroke prevention in an elderly community population with atrial fibrillation (the Birmingham Atrial Fibrillation Treatment of the Aged Study, BAFTA): a randomised controlled trial. Lancet. 2007;370:493-503.

40. Diener HC, Eikelboom J, Connolly SJ, Joyner CD, Hart RG, Lip GY, et al. Apixaban versus aspirin in patients with atrial fibrillation and previous stroke or transient ischaemic attack: a predefined subgroup analysis from AVERROES, a randomised trial. Lancet Neurol. 2012;11:225-31.

41. Tomaselli GF, Mahaffey KW, Cuker A, Dobesh PP, Doherty JU, Eikelboom JW, et al. 2017 ACC Expert Consensus Decision Pathway on Management of Bleeding in Patients on Oral Anticoagulants: A Report of the American College of Cardiology Task Force on Expert Consensus Decision Pathways. J Am Coll Cardiol. 2017;70:3042-67.

42. Connolly SJ, Ezekowitz MD, Yusuf S, Eikelboom J, Oldgren J, Parekh A, et al. RE-LY Steering Committee and Investigators. Dabigatran versus warfarin in patients with atrial fibrillation. $\mathrm{N}$ Engl J Med. 2009;361:1139-51.

43. Lip GYH, Keshishian A, Li X, Hamilton M, Masseria C, Gupta K, et al. Effectiveness and Safety of Oral Anticoagulants Among Nonvalvular Atrial Fibrillation Patients. Stroke. 2018;49:2933-44, doi: 10.1161/STROKEAHA.118.020232.

44. Reinecke H, Brand E, Mesters R, Schäbitz WR, Fisher M, Pavenstädt $\mathrm{H}$, Breithardt $\mathrm{G}$. Dilemmas in the management of atrial fibrillation in chronic kidney disease. J Am Soc Nephrol. 2009;20:705-11.

45. Marinigh R, Lane DA, Lip GY. Severe renal impairment and stroke prevention in atrial fibrillation: implications for thromboprophylaxis and bleeding risk. J Am Coll Cardiol. 2011;57:1339-48.

46. Shah M, Avgil Tsadok M, Jackevicius CA, Essebag V, Eisenberg MJ, Rahme E, et al. Warfarin use and the risk for stroke and bleeding in patients with atrial fibrillation undergoing dialysis. Circulation. 2014;129:1196-203.

47. Chan KE, Lazarus JM, Thadhani R, Hakim RM. Warfarin Use Associates with Increased Risk for Stroke in Hemodialysis Patients with Atrial Fibrillation. J Am Soc Nephrol. 2009;20:2223-33.

48. Wiesholzer M, Harm F, Tomasec G, Barbieri G, Putz D, Balcke P. Incidence of stroke among chronic hemodialysis patients with nonrheumatic atrial fibrillation. Am J Nephrol. 2001;21:35-9.

49. Chan KE, Edelman ER, Wenger JB, Thadhani RI, Maddux FW. Dabigatran and rivaroxaban use in atrial fibrillation patients on hemodialysis. Circulation. 2015;131:972-9. 
50. Siontis KC, Zhang X, Eckard A, Bhave N, Schaubel DE, $\mathrm{He} \mathrm{K}$, et al. Outcomes associated with apixaban use in patients with end-stage kidney disease and atrial fibrillation in the United States. Circulation. 2018;138: 1519-29.

51. ClinicalTrials.gov Identifier: NCT02933697. Compare apixaban and vitamin-K antagonists in patients with atrial fibrillation (AF) and end-stage kidney disease (ESKD).(AXADIA).

52. Holmes DR, Reddy VY, Turi ZG, Doshi SK, Sievert H, Buchbinder $M$, et al. Percutaneous closure of the left atrial appendage versus warfarin therapy for prevention of stroke in patients with atrial fibrillation: a randomised non-inferiority trial. Lancet. 2009;374:534-42.

53. Holmes DR Jr, Kar S, Price MJ, Whisenant B, Sievert H, Doshi SK, et al. Prospective randomized evaluation of the Watchman Left Atrial Appendage Closure device in patients with atrial fibrillation versus long-term warfarin therapy: the PREVAIL trial. J Am Coll Cardiol. 2014;64:1-12.

54. Reddy VY, Doshi SK, Kar S, Gibson DN, Price MJ, Huber K, et al. PREVAIL and PROTECT AF Investigators. 5-Year Outcomes After Left Atrial Appendage Closure: From the PREVAIL and PROTECT AF Trials. J Am Coll Cardiol. 2017;70:2964-75. 